American Journal of Applied Mathematics
$2021 ; 9(1): 20-30$
http://www.sciencepublishinggroup.com/j/ajam
doi: 10.11648 /j.ajam.20210901.14
ISSN: $2330-0043$ (Print); ISSN: $2330-006 \mathrm{X}$ (Online)

\title{
Reviving the Sphinx by Means of Constants - Codes in a Creative Space
}

\author{
Hristo Smolenov \\ Department of Navigation, Naval Academy, Varna, Bulgaria
}

Email address:

smolenov@abv.bg

\section{To cite this article:}

Hristo Smolenov. Reviving the Sphinx by Means of Constants - Codes in a Creative Space. American Journal of Applied Mathematics. Vol. 9, No. 1, 2021, pp. 20-30. doi: 10.11648/j.ajam.20210901.14

Received: March 2, 2021; Accepted: March 22, 2021; Published: March 30, 2021

\begin{abstract}
This paper deals with ancient codes embedded in world famous artefacts like the Sphinx and pyramids on the plateau of Giza. The same spatial codes are to be found materialized in the most ancient processed gold on Earth - the treasure trove of Varna Necropolis $(4600-4200 \mathrm{BC})$. Amazing is the match found between those two groups of artefacts, bearing in mind that the gold from Varna Necropolis is at least 2000 years older than the pyramids. But the prototype of their sacred measure - the so called royal cubit - emerges in the system of measures related to the famous Varna Necropolis. It is there that I have been able to identify the prototypes of universal constants like the Golden ratio and Pi. The synergy of those two constants is there for us to uncover - for instance Pi times the Golden ratio. It is the simplest one in an array of complex proportions which come into effect in regard to primeval gold artefacts as well as pyramids. Surprisingly, the synergy of Pi with an exotic variant of the Golden ratio allows for the inference of a specific number, which matches the Inverse Fine structure constant. For this inference the ratio between the Planck-length and the Light second will be a starting point. On the one hand is the minimum span in the Space-time continuum (the Planck length), and on the other hand - the distance covered by light in vacuum within one second of time (the Light second). The sacred measure of pyramids is the royal cubit comprised of 28 equal parts called "fingers". The length of the Great Sphinx comprises 140 royal cubits. It can be viewed as a supermeasure from which the sizes of the three main pyramids on the plateau of Giza obtain. Suppose we were to divide this supermeasure (the Sphinx-length) in 28 equal parts by analogy with the royal cubit being divided in 28 fingers. The result would be a span of 5 royal cubits. I maintain that (the Planck-length) times (10 to the power of 35) times (the Golden ratio number) yields 5 royal cubits, slightly longer than 0.523 meters each. Wishing to get closer to the actual length of the Sphinx, we might as well use a variant of the Golden ratio - the square root of (13/8 x 21/13) whereby 8, 13, 21 are consecutive Fibonacci numbers.
\end{abstract}

Keywords: Sphinx, Pyramids, Golden Ratio, Fibonacci Numbers, Light Second, Planck Length, Varna Necropolis Gold

\section{Introduction}

In this paper I am launching a hypothesis about the dawn of civilization. I maintain that a hidden super-culture from 6th and 5-th millennia BC had evolved to become Civilization One. The oldest processed gold on Earth from Varna Necropolis, Bulgaria (4600 - 4200 BC) provides evidence of the First system of knowledge encoded in pieces of art. [1]

Now that these masterpieces have been extracted from the oblivion, primeval knowledge lives again. Its standards of harmony keep fascinating us. Studying the most ancient processed gold on Earth is a true revelation. It unveils an astonishing interrelatedness of artefacts. As if someone had carefully chosen their size, looking for earthly standards rooted in cosmic relationships. What they have in common is the system of measure put in code.

How come the measures of length are the same? Answering this question, we have to transcend the study of ancient art and deal with universal constants instead. It takes us beyond artefacts and into the realm of numbers. A kind of a code comes into force based on the constants and the Light second as a Unit of measurement. The miracle of ancient creativity was due to intuition, precise measurement and logical insight. The outcome was the First system of knowledge in the history of civilizations: codes obtained as if by sharing genius 
with the Universe.

How do we know if archaeological finds unveil a lost civilization? It takes a conceptual discovery - a kind of logical password to the knowledge, hidden in artefacts. It provides a chance for us to focus the logic of creativity - the system of thinking behind material finds.

This opens up new prospects for decoding the intangibles of universal intelligence. Knowledge pervades the patterns of thinking at all times. It can convey the magic of creativity in terms of measures and proportions. Implemented in more than 3100 gold items is the most ancient processed gold on Earth the treasure trove of Varna Necropolis (4600 - 4200 BC). [2]

It comprises 6 kilograms of pure gold shaped in pieces of art which at the same time can serve as information carriers. The artefacts from Varna Necropolis are some 4000 years older than the time of Pythagoras. To regard them as information carriers means to bring out the logic of the First system of knowledge. It has been encoded in gold, copper, ceramic or stone objects. But what all of these have in common are the patterns matching cosmic entities, of course with the proper scaling. The numerical aspect of such correlations has first been upheld and highlighted in the views of Pythagoras. With him, I shall try and extend this approach to the study of numerical codes in Creative spaces.

\section{When Cosmos Meant Beauty}

It was Pythagoras who launched the idea of the so called "music of celestial spheres". This was the core of his teaching that Cosmos meant Beauty in the overall organization of the world. Cosmos was a state of affairs determined by the laws of Nature in tune with aesthetic criteria.

According to Pythagoras, those were put into code using numbers, measures and proportions. From this perspective, the world was a paramount system of proportions, a realm of self-organized numbers which represent the interplay of constants.

The artefacts from Varna Necropolis are some 4000 years older than the time of Pythagoras. To regard them as information carriers means to bring out the logic of the First system of knowledge. It has been encoded in gold, copper, ceramic or stone objects. But what all of these have in common are the patterns matching cosmic relationships.

Those who shaped the artefacts 7 millennia ago had no clue about of up-to-date science. And yet, contemporary notions can help unveil the Grand mystery of measures encoded in those artefacts. One can hardly believe that the correlations obtained are not merely coincidental. Yet the synergy of universal constants yields an enchanting interplay of proportions. It confirms that Pythagoras was quite right to consider the world as a system of proportions.

Studying artefacts from the distant past, we can try and unravel the mystery of knowledge as a self-reinforcing factor. It suggests that high intelligence had been out there to enable such remarkable creativity. The rise of ancient civilizations had been encouraged by an advanced source of inspiration.
This holds good for conceptual, as well as technological aspects of progress. Creative impetus had somehow been given to accelerate the pace, at which humanity was to develop. It happened by the Black Sea, on either side of the Balkan mountain. [3]

Its further development cast a mysterious spell on the later civilizations of Sumer and Egypt. Some of their achievements were inspired by a pre-existing system of knowledge pertaining to this enigmatic Super-culture. People back then had been able to grasp the intuitive meaning of some universal constants.

It occurs to me that the first encounter with the Golden ratio took place in Neolithic Zagora and Copper age Varna more than 7 thousand years ago. It was the time and place of Civilization One. Its messages were encoded in masterpieces of gold, copper, ceramic or stone. In light of those revelations, human creativity and Nature shared the same patterns of harmony...

Among them of the utmost importance is the Golden ratio. No wonder, the ceramic statue below was made in keeping with the divine proportion as early as 5-th millennium BC. It was found in a tell near to the city of Stara Zagora, Bulgaria, where the most ancient Neolithic dwellings in Europe have been unearthed. [4]

The statue of an ancient deity with two faces and four hands is a paradigm of the Golden mean implemented in art. Its characteristic proportion $g$ : $e$ approximates to the ratio of two consecutive Fibonacci numbers, namely

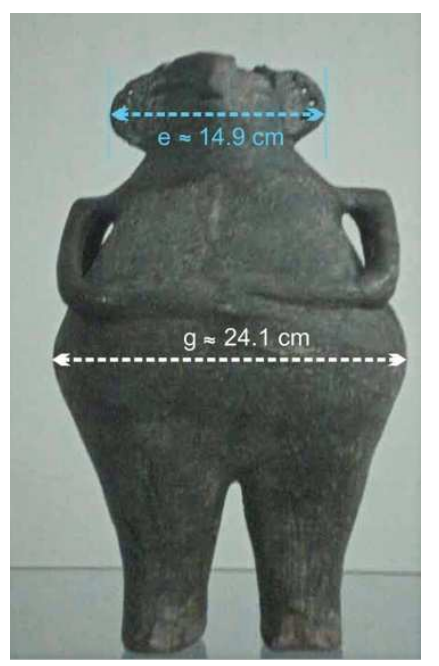

Figure 1. A ceramic statue of a deity from the 5-th millennium BC shaped in keeping with the Golden ratio, in terms of Fibonacci numbers. (55: 34).

$55: 34 \approx 1.617647$.

The sequence of Fibonacci numbers is comprised of the following members:

$1,1,2,3,5,8,13,21,34,55,89,144,233,377,610,987$, $1597,2584,4181$, and so on, and so forth.

The famous sequence uses a simple pattern: each of the Fibonacci numbers equals the sum of the two immediately preceding ones. Ratios of consecutive Fibonacci numbers can be regarded as variants of the Golden proportion. All such ratios are approximations of the unique number

$\varphi \approx 1.61803398875$, rounded off to $\varphi \approx 1.618$. This is roughly the value of the Golden mean, resp. the Golden ratio 
number. In the Renaissance, it was studied by Leonardo Da Vinci and referred to as "the divine proportion".

The sequence of Fibonacci numbers became known to the western science by the end of XII - beginning of XIII century, credit of Leonardo Fibonacci. He was a bright young scholar whose father had served with the Knights Templar. By the time Leonardo discovered the sequence, he had been studying the rate and proportions in which rabbits reproduced. So his discovery related to a natural process one might even say his inspiration sprang directly from Nature.

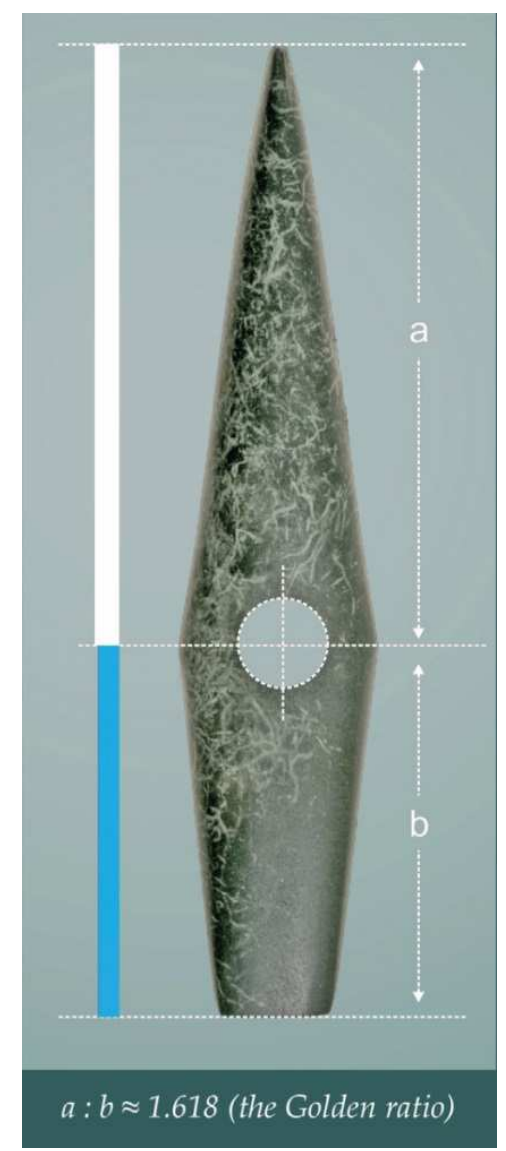

Figure 2. A stone axe from the Varna Necropolis (4600 - 4200 BC) perfectly proportioned ( $a$ : $b$ ) in keeping with the Golden ratio.

A variety of natural phenomena highlights characteristic features of the Golden ratio. It is generally associated with life and dynamics. The life of a star, too, unveils the divine proportion put into code with the help of Fibonacci numbers.

Look at the almost perfect sphere of raging plasma which is in Reality our Sun. It keeps transforming its mass into energy. So far, this has been happening for billions of years. Does it really make sense to measure the size of such a fireball? The answer is "yes", although in the long run the Sun will dramatically expand to engulf the bigger part of the Solar system. Hopefully, the catastrophe shall not take place before the elapsing of several billion years more.

For now, the Sun has a diameter of about 1.392 million km. [5] Using consecutive Fibonacci numbers I can derive a match for this numerical value. The pattern that goes with it yields a close approximation of the Sun's diameter measured by the astronomers. Given $\mathrm{S}=299792458 \mathrm{~m}$ and $\mathrm{T}=$ the square root of $(22 / 7)$ we arrive at

(S) $\times(\mathrm{T}) \times(34 / 21) \times(55 / 34) \approx 1.392$ million $\mathrm{km}$.

Here $(34 / 21)$ and $(55 / 34)$ are ratios of consecutive Fibonacci numbers standing for variants of the Golden cut. Therefore their product can be regarded as a variant of the Golden ratio squared.

In this way a characteristic feature of a cosmic entity - our Sun - has been presented in terms of a specific code. I have applied this approach to the diameters of the Earth and other terrestrial planets.

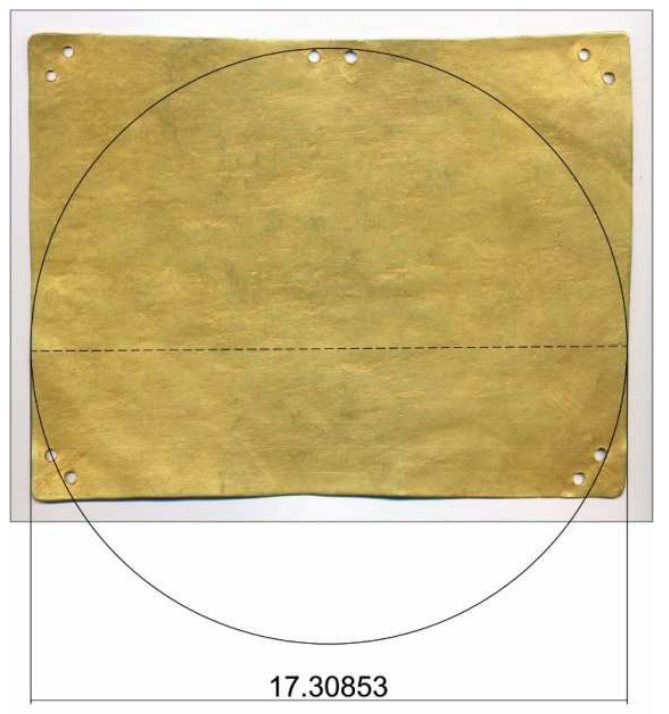

Figure 3. The bigger gold plate from Varna Necropolis, pertaining to the most ancient treasure trove of processed gold on Earth.

On the above diagram is a designated length $(\mathrm{G} \approx$ $17.3085 \mathrm{~cm}$ ) mapped on the scan of the bigger gold plate from Varna Necropolis (4600 - 4200 BC). Take a look at the formula

1 light nanosecond over the square root of 3

One billionth part of the Light second $=\mathrm{N}=$ one light nanosecond $=29.9792458 \mathrm{~cm}$.

Dividing $\mathrm{N}$ by the square root of (3), a measure of length is obtained. I shall call it the Golden Measure.

$\mathrm{GM} \approx 17.3085256 \mathrm{~cm}$.

Of course, this is an idealistic value if we should use it for the purpose of measuring objects. Nevertheless, it can be adequate for the sake of establishing important proportions. Here is a simple geometrical pattern involving the Golden measure in the following thought experiment.

Let $\mathrm{ABC}$ be an equilateral triangle, the height of which $(\mathrm{CH})=1$ light nanosecond. The tangent of 60 degrees $=$ the square root of 3 .

Dividing the light nanosecond $=29.9792458 \mathrm{~cm}$ by the tangent of 60 degrees, we obtain $\mathrm{AH}=\mathrm{HB} \approx 17.3085256 \mathrm{~cm}$. It corresponds to half the side of the equilateral triangle. So the Golden Measure obtained is equal to $\mathrm{AH}=\mathrm{HB}$, as shown on the diagram bellow. 


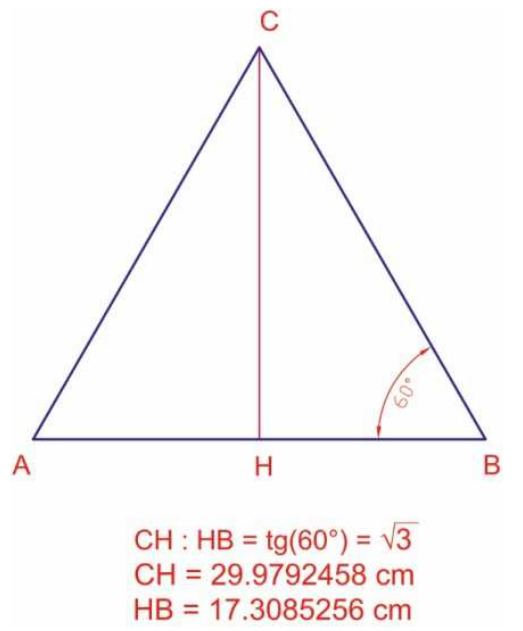

Figure 4. An equilateral triangle $A B C$ wherein the height $C H$ matches one Light nanosecond.

Although the Golden Measure is an idealistic value, the actual length of the big gold plate closely approximates to it. Is it due to the cosmic code?

Is it at all possible that actual dimensions of a celestial body, e.g. the Sun, or planet Earth, should be "deduced" from a Universal Unit of distance? Could it be that this Unit of distance equals the Light second = 299792458 m? Using a kind of a cosmic code, I shall try and substantiate this idea.

It is only natural that there should be some correlation between cosmic measures. The point is: an abstract Unit of length adequately applies to the real sizes of cosmic entities. The Sun's diameter has been approached in this way yielding a simple pattern of constants at work:

(S) times (T) times (34/21) times (55/34)

$=1391958.92 \mathrm{~km} \approx 1.392$ million $\mathrm{km}$

where (S) is the Light second; (T) stands for the square root of (22/7); (34/21) and (55/34) are consecutive ratios of consecutive Fibonacci numbers relating to the Golden ratio.
It would be a step forward to present a more or less similar pattern for the diameter of planet Earth. The challenge, in my opinion, would be to derive the equatorial circumference (resp. diameter) of the Earth from the interplay of the Golden Measure and the Golden proportion.

And again, in the style of the pattern above, I shall use ratios of consecutive Fibonacci numbers, more specifically the ratio $F=(89 / 55)$. My focus will be on the intriguing correlation between the main dimensions of two ancient artefacts. On the one hand is the gold plate from Varna Necropolis, home to the oldest treasure trove of processed gold (5-th millennium BC). [6] On the other hand is the Great Sphinx statue - approximately $73.34 \mathrm{~m}$ long.

It is high time we "revived the Sphinx" for the sake of building a bridge between lost knowledge from the very distant past and up-to-date notions about cosmic entities.

\section{Decoding the Great Sphinx}

It is now clear that the minimum length of the big gold plate from Varna matches the Golden Measure. Given F = (89/55), I am introducing the cube of this Golden mean, i.e. $(\mathrm{F} \times \mathrm{F} \times \mathrm{F})=\mathrm{F}^{3}=(89 / 55) \times(89 / 55) \times(89 / 55) \approx$ 4.23723. (One light nanosecond) over (the square root of 3 ) is the specific length which I refer to as the Golden measure.

$100 \times F^{3}$ times by the Golden measure equals

the length of the Sphinx, a value $\approx 73.34 \mathrm{~m}$.

So the theoretical length of Sphinx derives from the following two constants: 1) the Golden measure, obtained directly from the Light second; 2) the cube of the ratio $(89 / 55)$ of consecutive Fibonacci numbers. The pattern below shows a cosmic match found for the length of the Sphinx. It takes us beyond the ancient Riddle and into a new mystery. 100 times $(F \times F \times F) \times 17.3085256 \mathrm{~cm} \approx 73.34 \mathrm{~m}$

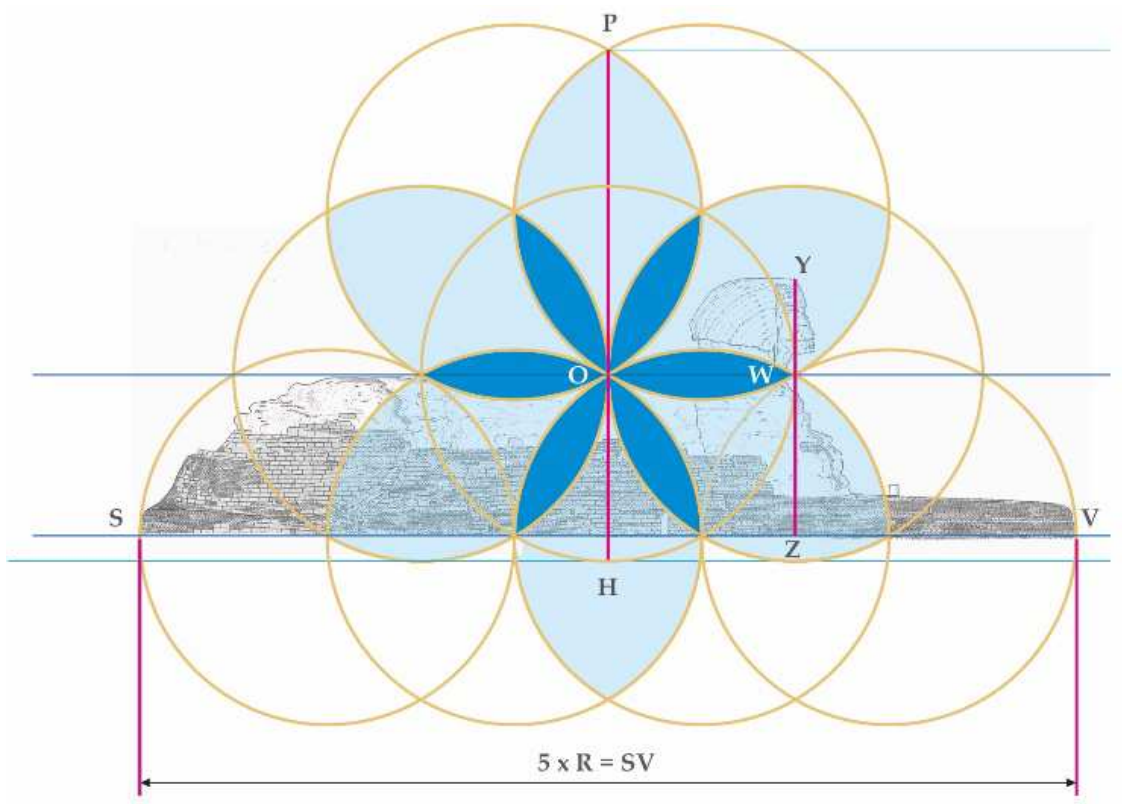

Figure 5. The silhouette of the Sphinx mapped on the Flower of Life. The height of the statue is $1 / 2$ PH while the base SV $=5 \mathrm{x}$ the radius. 

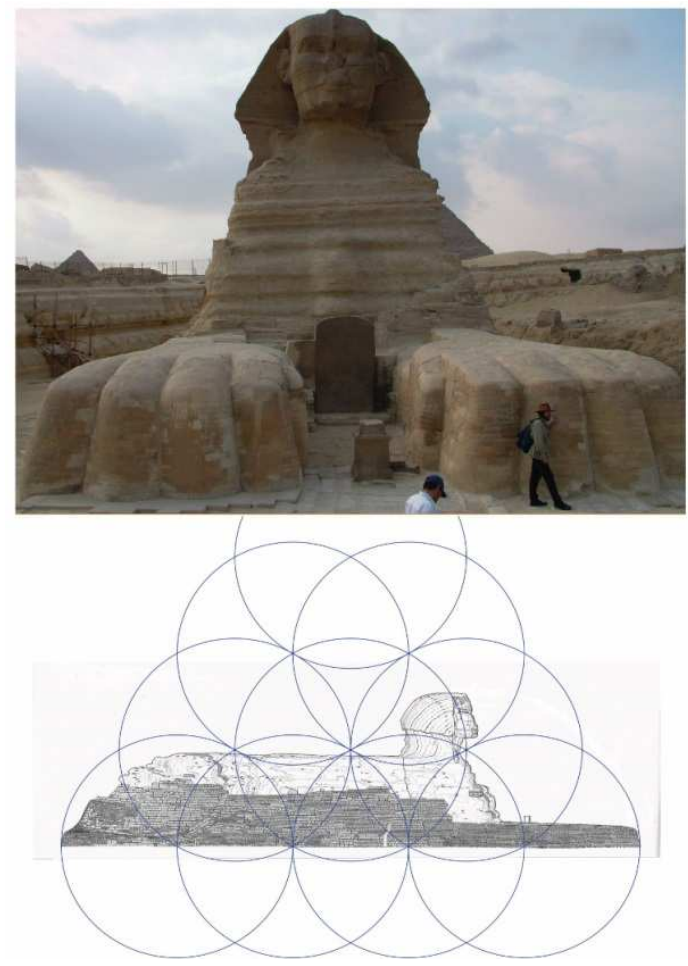

Figure 6. Front of the Sphinx facing east. It serves as the frame of reference for determining the centers of base of the three pyramids.

Since the distance 73.34 meters derives from the Light second, so does the equatorial circumference of the Earth. It is known to be $40075 \mathrm{~km}=1000000$ times $(40.075 \mathrm{~m})$. One-millionth part of our planet's circumference matches the characteristic length $\mathrm{PH}$ in the diagram of the Flower of Life below. Meanwhile the line SV matches the theoretical baselength of the Sphinx.

Here is a photo of Dr. Robert Schoch in front of the Great Sphinx. According to him, the statue is much older than what mainstream scholars have been suggesting. [7]

The statue is $20 \mathrm{~m}$ high, while the length of its base is a little over $73.3 \mathrm{~m}$. I shall try and map the sculptural profile of the Sphinx on the pattern of overlapping circles known as the Flower of Life. It is an ancient geometrical method of revealing proportions and drawing standard angles used for the sake of architecture, measuring land and even navigation.

Here the silhouette of the Sphinx has been outlined in a very precise manner due to the excellent book by Mark Lehner "The Complete Pyramids". [8] The diagram allows for an adequate study of the linear and angular proportions of the huge monolith.

Along these lines a number of surprising finds await us while "reviving" the Sphinx. As far as its actual dimensions are concerned, there is a real surprise in store for us. A match can be found for the equatorial circumference of the Earth, encoded in the size of the colossal statue. Mapping the sculptural profile of the Sphinx on the Flower of Life, we can show how this occurs.

The height of the statue $\mathrm{YZ}$ has been designed to match half of the line $\mathrm{PH}$ on the diagram. If $\mathrm{PH}$ equals $\approx 40.075 \mathrm{~m}$ (one-millionth part of the Earth's circumference), the line SV stands for the theoretical length of the Great Sphinx $\approx 73.34$ meters.

Summing up:

1. The Sphinx has been proportioned in keeping with the ancient geometrical pattern called the Flower of Life.

2. As far as proportions are concerned, the length of the statue matches 5 times the radius $\mathrm{R}$ of all the nine circles in this pattern.

3. The height of the Sphinx is designed to match half of the designated line PH painted red on the diagram below.

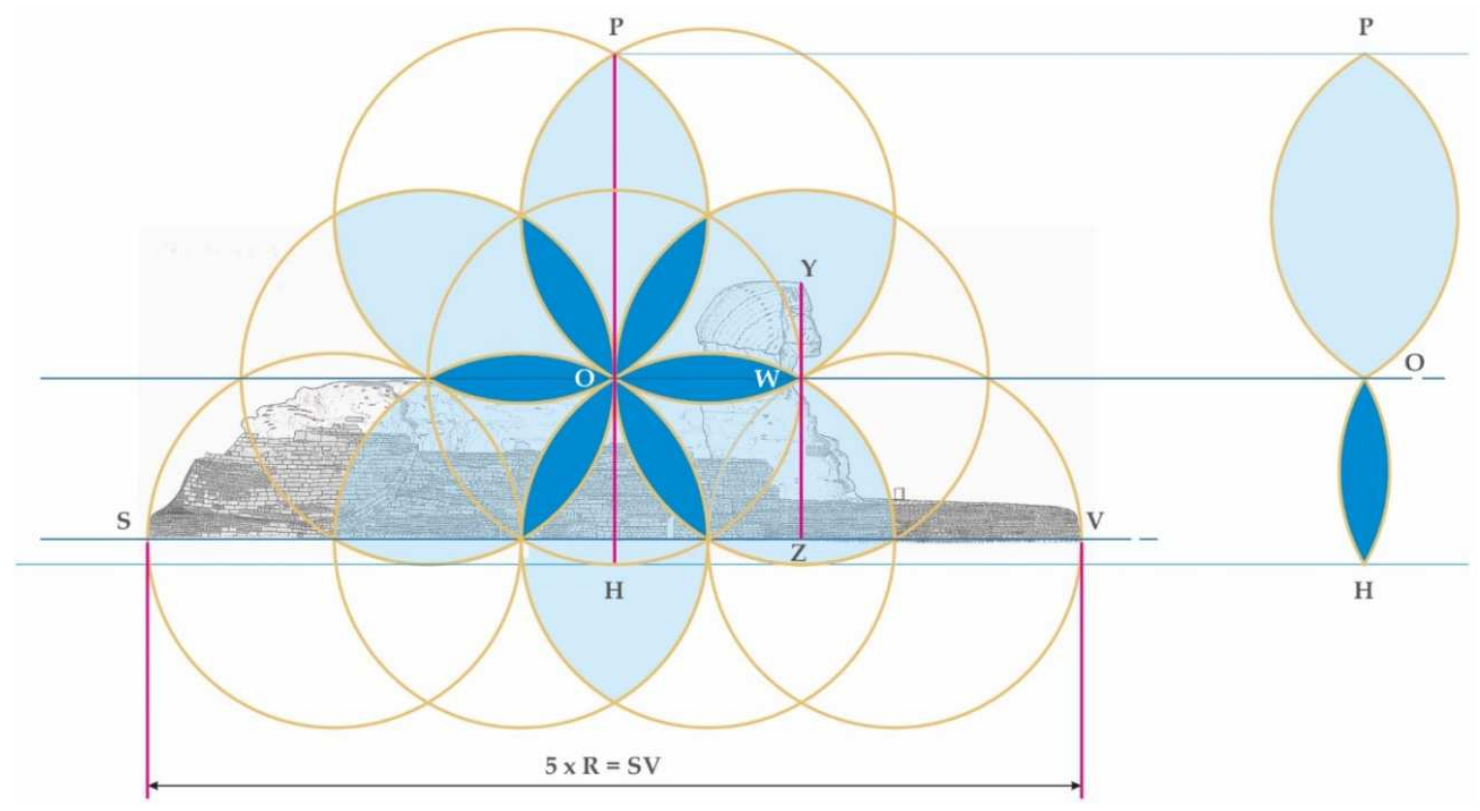

Figure 7. The Flower of Life pattern with two different sets of "petals". The outer is painted in light blue, while the inner is darker. The line PH equals the sum of the lengths of the outer + inner "petals" =PO +OH. Provided that PH is $40.075 \mathrm{~m}$, the line SV matches the actual length of the Sphinx $\approx 73.34 \mathrm{~m}$. 
4. PH is a characteristic length in this pattern. It equals the combined lengths of the outer and inner "petals" in the Flower of Life, so $\mathrm{PH}=\mathrm{PO}+\mathrm{HO}$.

5. The following equations hold true by design:

$\mathrm{YZ}=\mathrm{PH} / 2 ; \mathrm{YW}=\mathrm{HO} / 2 ; \mathrm{ZW}=\mathrm{PO} / 2$

The silhouetted Sphinx adequately represents the profile of the colossal statue. Still, it is but an image mapped on a diagram - an idealistic design with far reaching implications in the Reality.

6 . Let $\mathrm{YZ}$ be the height of the Sphinx $\approx 20 \mathrm{~m}$. So $\mathrm{PH}=2 \mathrm{x}$ $\mathrm{YZ}$ is $\approx 40 \mathrm{~m}$. This has been put into code, bearing in mind that the inner "petal" of the Flower matches the radius $\mathrm{R}=$ $\mathrm{HO}=\mathrm{WO}$. Assuming the outer petal PO is $(\mathrm{R} x$ the square root of 3$)$, the line $\mathrm{PH}=\mathrm{HO}+\mathrm{PO}$ represents $R \times(1+\sqrt{3})$.

If this corresponds to one millionth part of the Earth's equatorial circumference $(\approx 40.075 \mathrm{~m})$, then $S V$ stands for the distance $\approx 73.34 \mathrm{~m}$ which closely approximates to the actual length of the Great Sphinx. Is there no hidden message from the remote past encoded in this legendary monolith?

Mythology has it that the living Sphinx kept asking one and the same question. It was all about a mysterious animal that walked on four legs in the morning, then on two legs at noon and on three legs in the evening. Numbers 4, 2, 3.

Needless to say, this can be seen as a metaphor. No animal whatsoever would be the answer to this ambiguous question. Not even the human being, as was the answer of the mythical Oedipus King. According to him, human beings move on their four limbs as babies, then on two legs as adults. Eventually, when they are old, they walk "on three legs", if a walking stick is to be taken into account. Such a solution is too simple-minded even for the average myth-consumer. With all due respect to legends, this one in particular looks like a soap opera. In my opinion, the message of the Great Sphinx is about numbers, not mysterious animals.

The numbers 4, 2, 3 in order of appearance convey some hidden meaning... Given the sequence

4 in the first place = "in the morning"

2 in the second place = "at noon" and

3 in the third place $=$ "in the evening"

we may think of the number 423. In my opinion, it bears reference to the cube of the Golden ratio represented as one hundred times the product of three ratios of consecutive Fibonacci numbers. For example,

100 times $(21 / 13)$ times $(34 / 21)$ times $(55 / 34) \approx 423$

The rest is a mnemonic - a way of remembering things by repeating them. In this mnemonic form ancient wisdom has endured. It must have sounded like an anecdote, so people should keep telling it, not necessarily understanding its hidden meaning.

The cube of the Golden ratio was the key to the Riddle of the Sphinx, but also to the abstract structure of the huge statue. Its dimensions turned out to be in tune with the gold standard from Varna. This system of measures had survived the turbulences of two millennia, so as to reappear in the Sphinx and pyramids. What a rebirth of knowledge!

Avoiding the exaggerations of wishful thinking, at least we have to admit the following. Some patterns in use by ancient thinkers look as though they were inspired "from above". To put it in a different way, those patterns look as if they were obtained by way of highly strung communication in the process of sharing genius with the Universe.

After all, why deny the option of a universal intelligence pertaining to the ultimate distributed system known as Nature? Distributed systems generate additional capacities for wielding knowledge and self-organized behavior. It takes both logic and intuition to use these capacities, drawing upon the immense intelligence that surrounds us.

Here is a fact relating to the characteristic length of the Great Sphinx. Let $(\mathrm{AC} \approx 73.34 \mathrm{~m})$ be the diameter of a circle and at the same time diagonal in the square ABCD. Then each side of the square equals 100 Nippur cubits, the equivalent of $\approx 51.86 \mathrm{~m}$. So the length of the Sphinx is 100 times the exact Nippur cubit (the sacred measure of Sumer) multiplied by the square root of 2 .

How come the Sumerian standard is encoded in the size of the huge statue on the plateau of Giza. By the way, in the language of ancient Sumerians the name Giza means "Throne". In reality, the plateau rises like a throne over the much lower lands irrigated or flooded by the Nile...

I maintain that the Sphinx was carved from the bedrock not just for the sake of decoration. This accomplishment of sacred geometry was designed as a tool of knowledge. The duality of sacred measures - Sumerian and Egyptian - implies that both of them might derive from one and the same prototype. The system of measures born in Copper age Varna (middle of 5-th millennium BC) might have provided such a prototype. As for the Sphinx statue, it might as well pertain to Civilization One. Hence the role of the Golden measure matching the minimum length of the gold plate in the model.

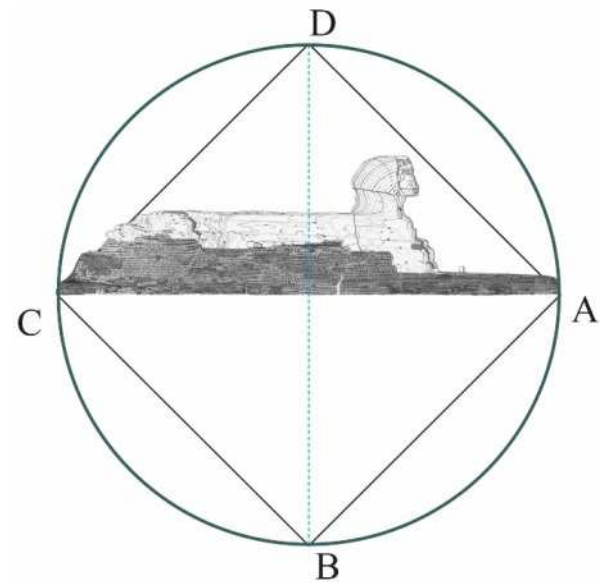

Figure 8. If the Sphinx-length $73.34 \mathrm{~m}$ is the diagonal of the square, the sides $A B=B C=C D=D A=100$ Nippur cubits, each of them $\approx 0.5186 \mathrm{~m}$.

Given $F^{3}=(89 / 55) \times(89 / 55) \times(89 / 55)$, a pattern obtains yielding the Sphinx's theoretical length $=$ $100 F^{3} x$ (the length of the gold plate) $\approx 73.34 \mathrm{~m}$.

The above pattern highlights the role of constants in the making of the legendary statue. According to Herodotus, the Great pyramid was 280 royal cubits high, while the side of its base was 440 royal cubits long. [9] 


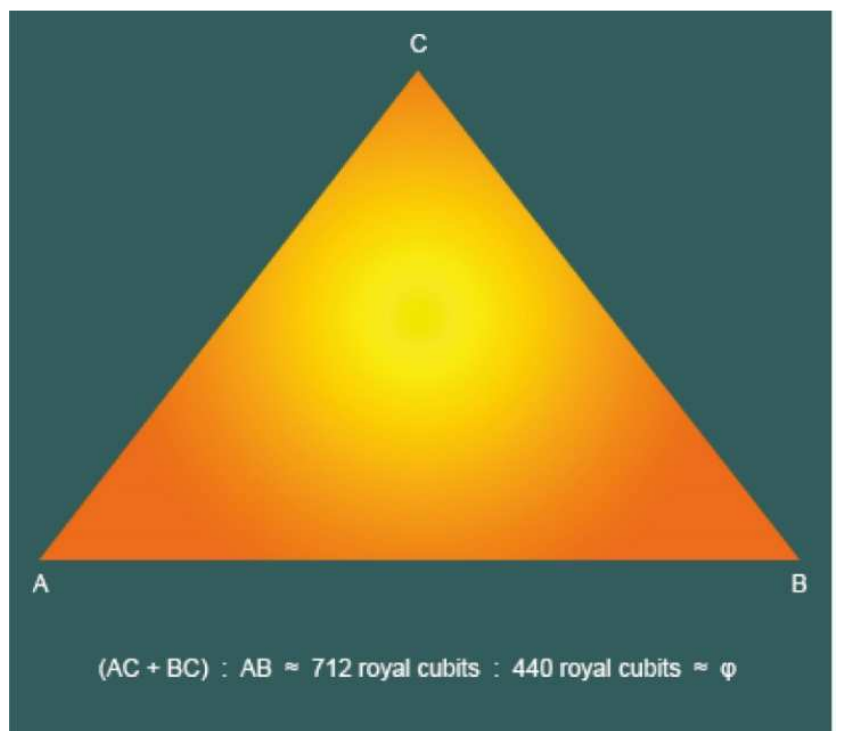

Figure 9. A model of the vertical cross-section of the Great pyramid. The sum of the two slopes over the base approximates the Golden ratio.

Let us relate those two values to the theoretical length of the Sphinx statue, estimated approximately 140 royal cubits.

440 royal cubits over 140 royal cubits $=22 / 7=\Pi$

280 royal cubits over 140 royal cubits $=2$

So, the base-to-height ratio of the Great pyramid is $(22 / 14)$

$=(\Pi / 2)$. As for the slopes-to-base proportion, it is $(\mathrm{AC}+\mathrm{BC})$ over $\mathrm{AB} \approx 712$ royal cubits over 440 royal cubits $=89 / 55$

The synergy of these main proportions makes the Great pyramid look so enigmatic and impressive.

Using a simplified model of the vertical cross-section of the famous pyramid can shed light on the interplay of its main proportions. For this purpose, I shall once again resort to the strict but flexible tools of the Fibonacci sequence.

712 royal cubits over 440 royal cubits $=\mathrm{F}=89 / 55$

712 royal cubits over 140 royal cubits $=(22 / 7) \times(89 / 55)$.

The sum of the two slopes is rounded off to 712 royal cubits, i.e. each of them is $\approx 356$ royal cubits long. This is obtained by way of the theorem of Pythagoras, given the half base $=220$ royal cubits and the height $=280$ royal cubits.

More precisely, $\mathrm{AC}=\mathrm{BC} \approx 356.09$ royal cubits, therefore $\mathrm{AC}+\mathrm{BC} \approx 712.18$ royal cubits corresponds to the sum of the two slopes. So,

712.18 royal cubits over 140 royal cubits $\approx$

$\Pi \times \mathrm{F}_{1} \approx(22 / 7) \times 1.61859$

Here $F_{1}$ is no arbitrary approximation of the Golden ratio. It has been derived from the sequence of Fibonacci numbers in the following way.

The ninth root of the ratio (610 over 8$)$ is equal to $1.618593384 \ldots$ It can be rounded off to 1.61859 . Bear in mind that 610 and 8 are Fibonacci numbers, 610 being the ninth member after 8 in the Fibonacci sequence. So the product of 9 ratios of consecutive Fibonacci numbers is

$(13 / 8) \times(21 / 13) \times(34 / 21) \times(55 / 34) \times(89 / 55) \times(144 / 89) \times$ $(233 / 144) \times(377 / 233) \times(610 / 377)=(610 / 8)$

The ninth root of this product yields the related variant of the Golden proportion. Multiplied by (22/7), it produces the complex proportion $\Pi$ x $\mathrm{F}_{1} \approx(22 / 7) \times 1.61859$.

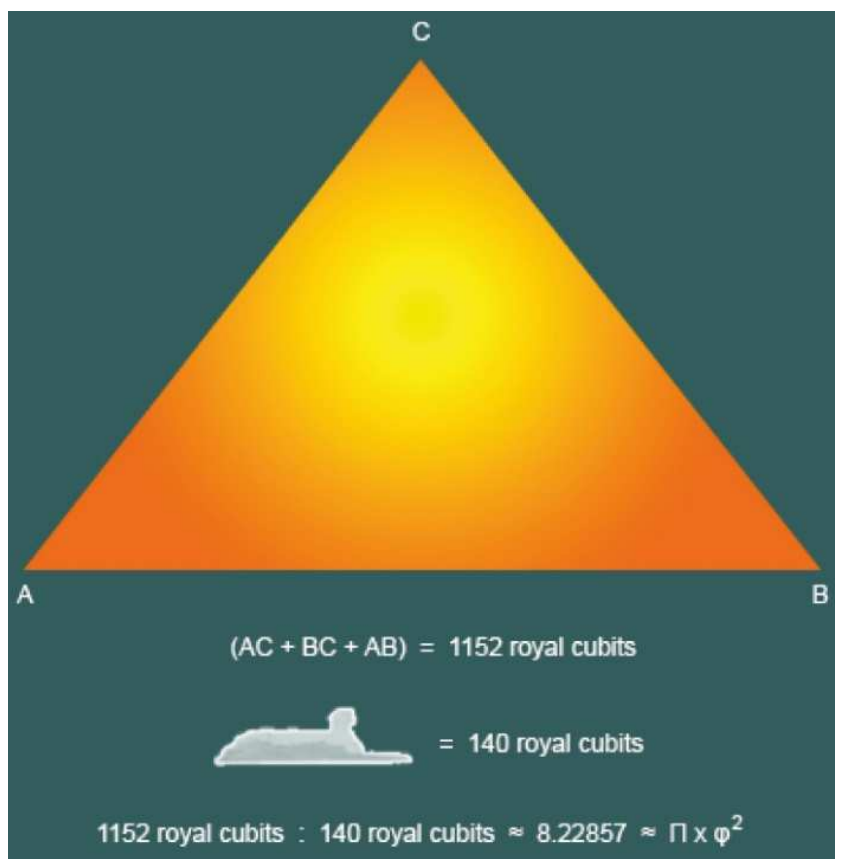

Figure 10. The numerical value of the proportion between the perimeter of the pyramid and the length of the Great Sphinx.

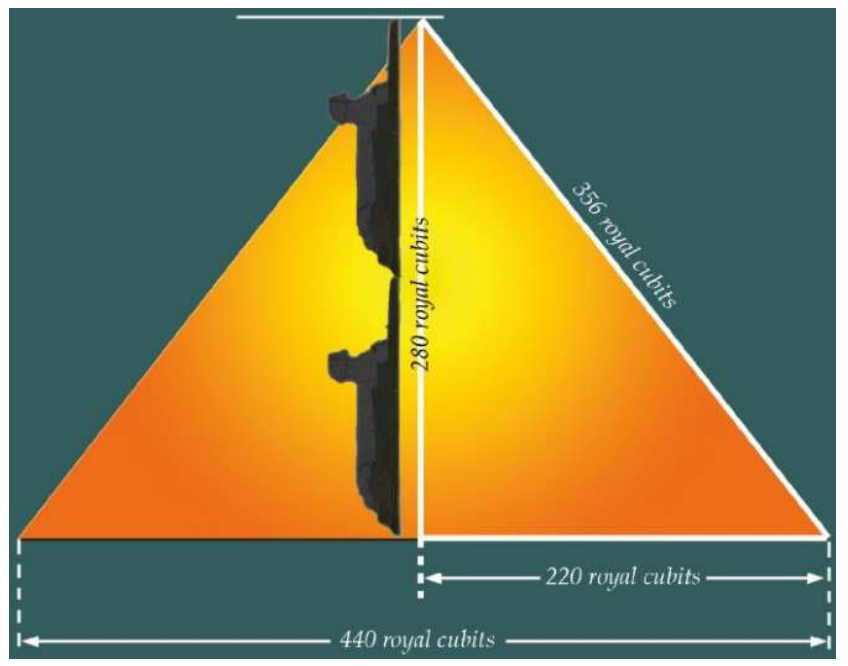

Figure 11. The height of the Great pyramid approximating twice the length of the Sphinx statue.

The proportion between the perimeter $(\mathrm{AC}+\mathrm{BC}+\mathrm{BA})$ and the side-of-the base is

$(1152$ royal cubits $)$ over $(440$ royal cubits $)=2.6181818 \ldots$

This number obtains from the product $(89 / 55) \times(144 / 89)$ wherein 55, 89, 144 are consecutive Fibonacci numbers. More precisely, the proportion between the perimeter (1152.18 royal cubits) and the length of the Great Sphinx approximates to $\left(\Pi \times \mathrm{F}_{2} \times \mathrm{F}_{2}\right)$. It is the synergy of constants, namely (22/7) and the squared variant of the Golden ratio.

Using the Sphinx-measure as a standard, we can represent the theoretical height of the Great pyramid. As Herodotus has observed, its equals 280 royal cubits. This means 2 x 140 royal cubits, i.e. twice the length of the Sphinx statue.

The Sphinx-measure also matches the distance between the Subterranean chamber and the floor of the so called 


\section{King's chamber. [10]}

The exact position of the Great sphinx and its length are two arguments, a function of which is the pyramid's centerof-base. Its distance from the Sphinx is the hypotenuse of a triangle with sides 660 royal cubits and 840 royal cubits respectively.

Six times the Sphinx-measure $=6 \times 140$ royal cubits $=840$ royal cubits. While 3 times 140 royal cubits times $(22 / 14)=$ 660 royal cubits.

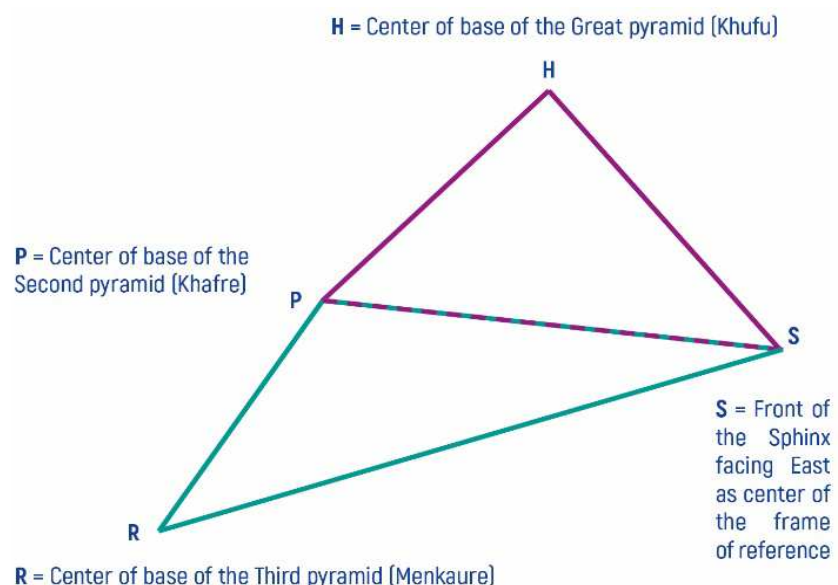

Figure 12. The base-centers of the three pyramids determined by the exact position of the Sphinx. It probably preceded them in time.

In my opinion the patterns of alignment of the Sphinx and pyramids in Giza are the outcome of logical and geometrical notions, not just star-gazing analogies. Along these lines the popular "belt of Orion" analogy is a fine astronomic metaphor. It is a poetic way of expressing intuitive hunches but there are logical and geometrical reasons for the actual alignment of the three pyramids. With all due respect for the Orion hypothesis by Robert Bauval [11] I wish to add a point about the purely geometrical grounds for this peculiar alignment. The diagram above shows the exact positions of Khufu, Khafre and Menkaure in regard to the Sphinx. They are determined by the place of the Great Sphinx on the plateau of Giza. The distance between its front and the basecenters of the main pyramids have been fixed in keeping with the following measures.

$\mathrm{SH}=$ the hypotenuse of a right angled triangle, its sides being

a) 840 royal cubits $=6 \times 140$ royal cubits;

b) 660 royal cubits $=3 \times 140$ royal cubits $\times 22 / 14$.

$\mathrm{SP}=3 \times \mathrm{AB}=$ three times the side of the base of the Great pyramid.

$\mathrm{SR}=3 \times \mathrm{AC}=$ three times the diagonal of the same pyramid.

\section{Codes in a Creative Space}

The diagram above shows the difference between the theoretical height of the Great pyramid (approximately 146.6 $\mathrm{m}$ ) and its actual height (roughly $138.75 \mathrm{~m}$ ). This difference is due to the missing apex. But in my opinion the actual height conveys a message. It involves to the size of the so called "finger" $=1.875 \mathrm{~cm}$, as part of the royal cubit.

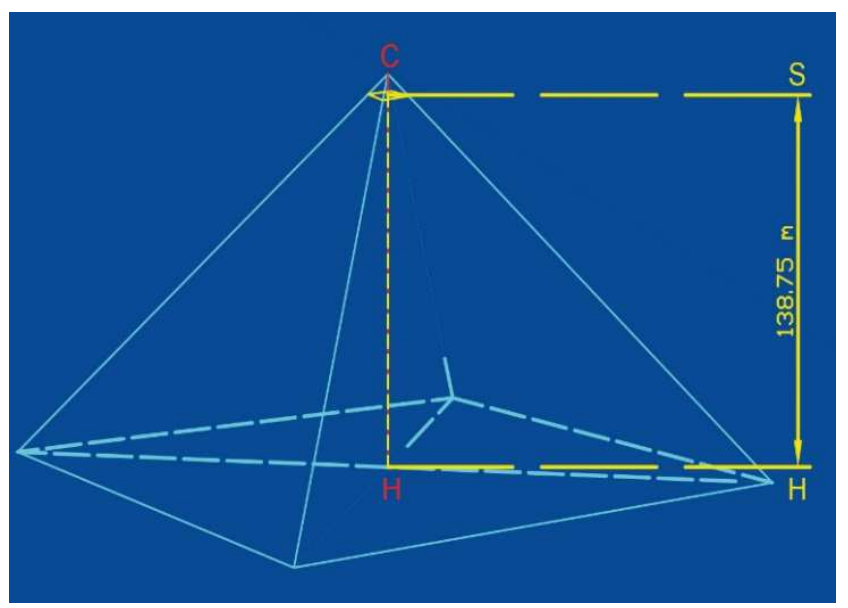

Figure 13. The real height of the Great pyramids is $138.75 \mathrm{~m}$. There might be a message encoded in this dimension of the huge building.

Let the number 137.035999 be the numerical value of the Inverse Fine structure constant - a notion from physics which deserves special attention. Multiplying it by the combined length of 54 fingers, we closely approximate the actual height of the pyramid.

$$
(137.035999) \times 54 \times(1.875 \mathrm{~cm})=13874.89 \mathrm{~cm}
$$$$
\approx 138.75 \mathrm{~m}
$$

Is this just a coincidence, or is there anything deeper to it?

The numerical value of the Inverse Fine structure constant is $\approx 137.035999$. If it had not been for this value, the world as we know it would not at all be in existence. To put it in another way - it is this constant that permeates the Reality as a whole - it builds the Universe as a distributed system of interrelated entities. Even a slight deviation from this value would mean the end of Cosmos and the triumph of Chaos.

I maintain that a match for the Inverse fine structure constant can be derived from the synergy of four constants. The starting point for this inference is the ratio between two remarkable measures of distance. On the one hand is the Planck length, which belongs to the system of the so called Natural units.

Its value is a little less than $1.616229 \mathrm{~m} \mathrm{x} 10$ to the power of (minus 35). The Planck length stands for a sort of an indivisible distance. It is the minimum length that still preserves the status of the Space-time continuum. On the other hand is the Light second in its capacity as Universal unit of distance.

The ratio of those two fundamental measures will be multiplied by $\pi / 2$ times an exotic variant of the Golden proportion. The latter obtains from the sequence of Fibonacci numbers as shown below.

The thirtieth root of the above product can be viewed as a paradigm of the Golden proportion. In this way we obtain

$F \approx 1.618201758$

Take a look at the formula below. To the left in the equation is the Inverse Fine structure constant $\approx 137.035999$. To the right is a product representing the following synergy: 
(The Planck length $\approx 1.61622875 \times 10^{-35} \mathrm{~m}$ ) over (the Light second $\mathrm{S}=299792458 \mathrm{~m})$ multiplied by $(\pi / 2$ times $\mathrm{F})$ times (10 to the power of 45 ).

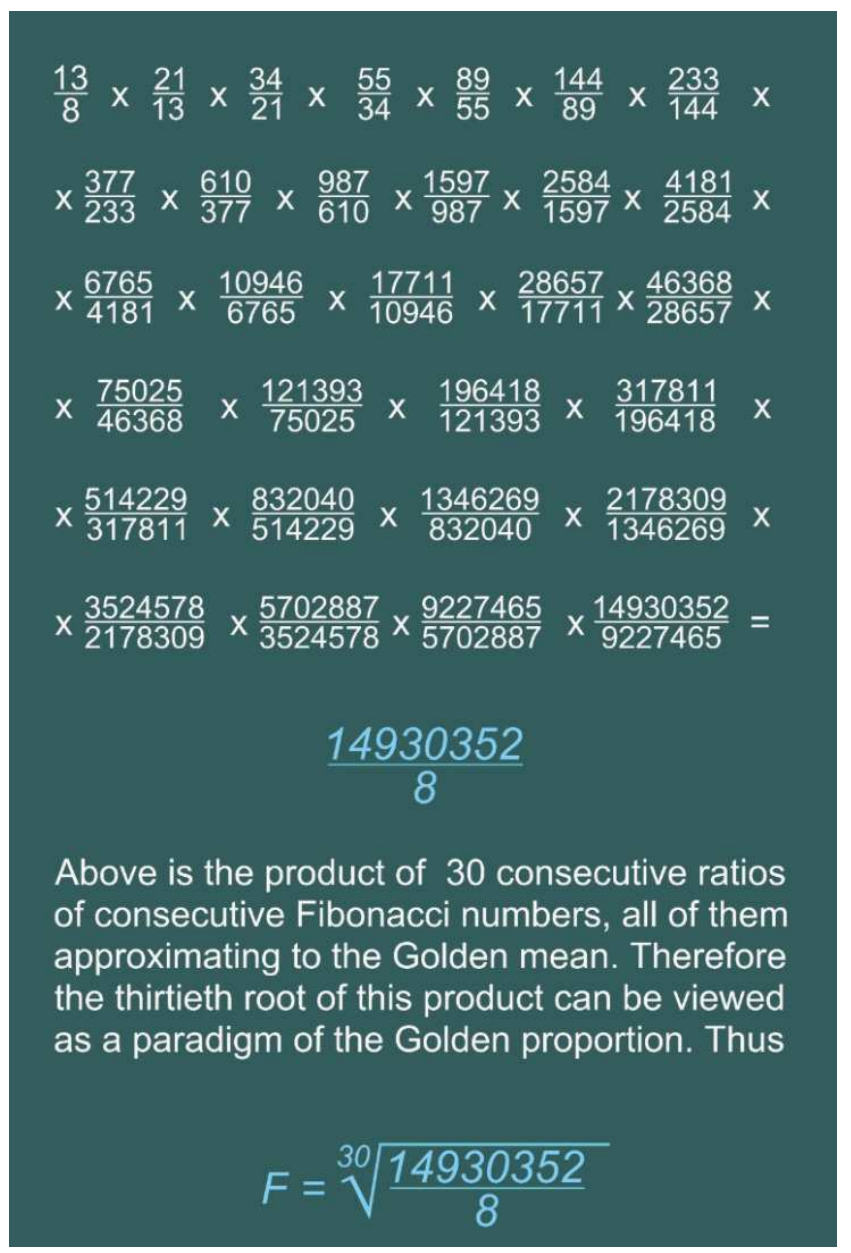

Figure 14. Here $F$ is a variant of the Golden mean. It is important for deriving the exact value of the Inverse Fine structure constant.

$$
1 / \alpha=(L p / S) \times(\pi / 2 \times F) \times 10^{45}
$$

Here $(\mathrm{Lp} / \mathrm{S})$ is the ratio between the Planck length and the Light second; $F$ is the exotic variant of the Golden mean derived from the consecutive ratios of consecutive Fibonacci numbers above. It helps derive the numerical value of the Inverse Fine structure constant $\approx 137.03599913$.

R. Feynman regarded it as "ONE OF THE GREATEST DAMN MYSTERIES OF PHYSICS: A MAGIC NUMBER THAT COMES TO US WITH NO UNDERSTANDING BY MAN". [6]

According to Feynman, it was impossible to logically obtain this number. But I have been able to prove the opposite, deriving a match for the Inverse Fine structure constant from the synergy of four other constants in a relatively simple equation. In this formula the product $(\pi / 2$ times F) represents a synergy of two universal constants, one of them being the Pi, while the other stands for a variant of the Golden mean as shown on Figure 14.

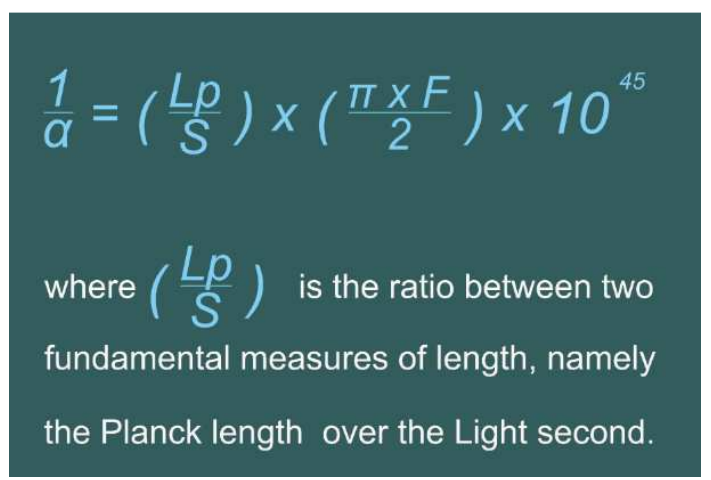

\section{Conclusion}

How come dimensions of artefacts from three different epochs are so remarkably correlated? Is there no logical clue to this chronological paradox? Simple patterns obtain along these lines building bridges between great achievements of different civilizations.

We are surprised to find out that masterpieces from Copper age Varna (6-th - 5-th millennia BC) are amazingly coherent with the giant monuments from Giza (3-rd millennium BC) as far as their measures of length are concerned. Even bigger is the surprise when research takes us beyond earthly creations and into spatial relationships between cosmic entities. This astounding coherence is in full sway as regards the characteristic dimensions of celestial bodies. Even the distances between them reveal some patterns of harmony.

For instance, variants of the Golden ratio can help unravel the mystery of the lunar distance. It is the average distance from our planet to the Moon, estimated $384402 \mathrm{~km}$. Onemillionth part of the lunar distance is rounded off to $384.4 \mathrm{~m}$. Let $\mathrm{F}=34 / 21$ be a variant of the Golden mean in terms of Fibonacci numbers. Given $F^{2}=F$ x F $=(34 / 21$ x 34/21), we can calculate

$384.4 \mathrm{~m}$ over $(\mathrm{F} \times \mathrm{F}) \approx 146.644 \mathrm{~m}$

This is a match for the theoretical height of the Great pyramid! Here is a correlation between three focal points.

1) A gold plate from Varna Necropolis.

2) An enormous pyramid on the plateau of Giza.

3) The Lunar distance as a specific cosmic relationship. At the core of this correlation are variants of the Golden mean obtained from ratios of consecutive Fibonacci numbers. More specifically, the lunar distance has been decoded as a synergy of constants. The same holds true for the length of the orbit path of the Moon moving around the Earth. It has been estimated $\approx 2413402 \mathrm{~km}$. Let us divide this distance by the following complex constant:

$$
\pi \times(89 / 55)^{3}
$$

The result obtained is $181300 \mathrm{~km}$. This is no random span at all. Rather, it is a kind of a cosmic code by means of which we can try and represent important spatial relationship. The relation between the perigee and apogee of the Moon orbiting the Earth can be decoded in the following way. A right angled triangle $\mathrm{ABC}$ with sides 2: 1 is the basic model. Let the shortest side $\mathrm{AC}$ be $181300 \mathrm{~km}$. The longer side $\mathrm{AB}$ 
would be equal to $2 \times 181300 \mathrm{~km}=362600 \mathrm{~km}$, while the hypotenuse $\mathrm{BC}$ equals the square root of (5) times 181300 $\mathrm{km} \approx 405400 \mathrm{~km}$.

This is a match for the apogee, i.e. the point furthest from the Earth that the Moon reaches while moving around our planet. As for the value $362600 \mathrm{~km}$, it corresponds to the perigee, the nearest point to the Earth that the Moon reaches while orbiting our planet.

A simple geometrical interpretation helps decipher an aspect of the Pythagorean doctrine.

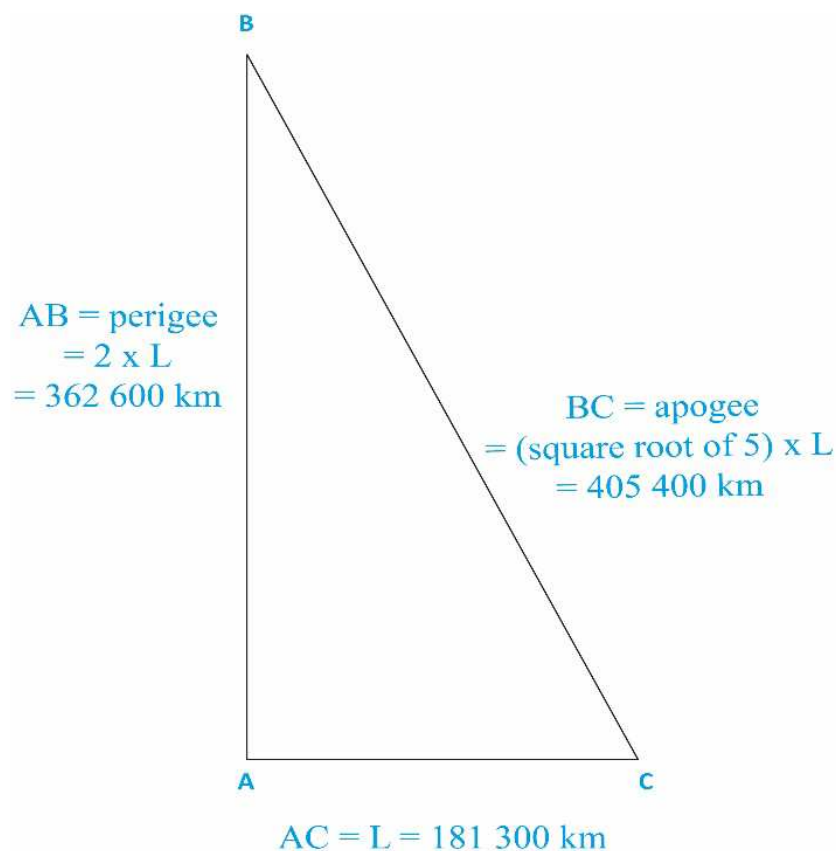

Figure 15. This right angled triangle provides an exact model for the relationship between the perigee and the apogee of the Moon orbiting the Earth. The distance $L=181300 \mathrm{~km}$ derives from the orbit path of the Moon moving around the Earth. The distance $2413402 \mathrm{~km}$ being divided by $\pi x$ $(89 / 55)^{3}$ yields $181300 \mathrm{~km}$. It is the base AC which determines the two other sides in the triangle.

In the Reality itself there are aspects of harmony which Pythagoras used to denote as the music of celestial spheres. It was no music for the ears, but rather - a realm of complex proportions in tune with aesthetic as well as logical criteria.

What could be the reason why ancient masters were so poetic about Nature, while remaining meticulous about precision? In my opinion, they needed the accuracy for the sake of honoring what they perceived as cosmic harmony. It looked as though people had been invited to take part in a ritual of universal creativity. This was their first encounter with a Cosmic code shedding light on what struck the imagination of people thousands of years ago. A match was there for them to find and a secret of Nature theirs to decode.

It all began with Zeno's paradoxes relating directly to the intangible reality of Space. Zeno refuted the simplistic views on the structure of physical Space as infinitely divisible into parts smaller than the whole. [13] Meanwhile the ancient atomists following Democritus placed atoms and space at the beginning of all things. They upheld the two categories as axiomatic pillars of the Universe. Long before their time, far beyond the horizons of prehistory primeval thinkers had been aware of the mystery of spatial relationships. They were keen on measuring them with the help of standards. Amazingly enough, those standards had intriguing cosmic matches.

This is how the Nippur cubit in Sumner and the royal cubit in Ancient Egypt came into being. But they had a common prototype in use at least 2000 years earlier. It was the achievement of Civilization One - the First civilization of knowledge born by the Black Sea, on either side of the Balkan mountain. [14]

Primeval masters chose to uphold precision out of respect for the laws of Nature. And at the same time it was a way of showing the self-esteem of creative minds. They felt like sharing the responsibility for the Grand harmony of the world. Theirs was an awe inspiring wish to match celestial beauty by shaping earthly masterpieces. [15]

While the forces of Nature were regarded as sovereign in this process, humans perceived themselves as junior partners. This required high accuracy on their part in order to honor the creative partnership between human beings and universal intelligence. But material instruments in the remote past were not up to such a momentous task. So the accuracy of thinking had to compensate for the lacking accuracy of tools. Here mathematics and logics came to the rescue. It was at this point that primeval thinkers grasped the idea: Space was the format of the Reality - the factor responsible for the arrangement, design, even the organization of things that took place in it.

Space Matters!

If properly decoded and taken into account, the intangible reality of Space opens up new prospects for creativity. Ancient atomism provides ample evidence along these lines. But it has also a kind of ontological meaning to it, relating to the study of existence, not just the possibility of knowing things.

Here I have launched a set of ideas to the effect that Space is the format of universal intelligence, too. It has the virtual capacity for self-design. To put it in another way, a creative space is capable of self-organization through the interplay of universal constants. The synergy of Pi with variants of the Golden ratio is a paradigm of this virtual interplay. But when it comes to the Light second as the unit of distance, extremes meet and a virtual capacity can be upgraded to objective reality. No wonder that a match for the Inverse fine structure constant has been derived in this paper using a purely spatial relationship - the Planck length over the Light second.

Here is what John Baez wrote in 2011: "certain constants don't depend on the units we use - these are called dimensionless constants. Some of them are numbers like pi, e, and the golden ratio - purely mathematical constants which anyone with a computer can calculate to as many decimal places as they want. But others - at present - can only be determined by experiment. These tell us facts about nature that are completely independent of our choices of units. The most famous example is the fine structure constant... experiments show that it's about 1/137.35999. Nobody knows why it equals this. At present, it's a completely mysterious raw fact about the Universe!' [16] 
To grasp this is but the first step in a long path of arguments. They substantiate my hypothesis to the effect that a kind of logical resemblance exists between Space and Matter. As far as their structure is concerned, there are limits to divisibility. Beyond these limits, qualitative changes occur and new realms of the Reality come into force.

For example, the Planck length is to be regarded as the minimum span in Space-time, a distance which defies further divisibility into parts smaller than the whole. Beyond it, quantum properties are expected to prevail changing the very notion of space-time in physics.

I feel tempted to introduce the term "geometron" to account for this minimum distance corresponding to the Planck length as one of the so called Natural units. The system of Natural units, proposed by Max Planck has a far reaching impact on future scientific developments. And with it comes a new outlook on the interrelation between the key notions describing Space-time and Energy-Matter.

So it is not just a play on words for me to eventually claim that a Creative space does matter. It is the synergy of universal constants that permeates the Reality, accounting for its space-temporal, as well as field-material aspects. The related interplay of fundamental proportions appears to be what Pythagoras denoted as "the music of celestial spheres".

\section{References}

[1] Hristo Smolenov, Aurolithic Patterns of Synergy: Variations on the Golden Ratio Theme. Symmetry: Culture and Science, Vol. 28, No. 4, 2017.

[2] New Perspectives on the Varna Cemetery (Bulgaria) - AMS Dates and Social implications. Antiquity, 81, 2007.

[3] Hristo Smolenov and Hristo Michailov, The Lost Aurolithic Civilization. Codes from a Black Sea Atlantis, Sofia, 2010.
[4] Hristo Smolenov, Zagora - Varna, the Hidden Superculture. Institute of metal science, equipment and technology, Bulgarian Academy of Science. Sofia, 2012.

[5] Sir Patrick Moore, Atlas of the Universe. Sixth edition. Philip's, 2007. Reprinted 2010.

[6] Prince Mikasa, in: The First Civilization in Europe and the Oldest Gold Treasure in the World - Varna, Bulgaria. Nippon Television Network Cultural Society, 1982.

[7] Robert Schoch, Forgotten Civilization. The Role of Solar Outbursts in Our Past and Future, Inner Tradition, 2012.

[8] Mark Lehner, The Complete Pyramids, Thames and Hudson, 1997; Courtesy: Mark Lehner, The Complete Pyramids. The American University in Cairo Press, for the precisely delineated silhouette of the Sphinx.

[9] Herodotus, Histories. Cambridge, Massachusettes, Harvard University Press, 1946.

[10] The Seventy Great Mysteries of Ancient Egypt, edited by Bill Manley, Thames and Hudson, London, 2003.

[11] Robert Bauval and Adrian Gilbert, The Orion Mystery, Heinemann, 1994.

[12] Richard P. Feynman, The Strange Theory of Light and Matter, Princeton University, 1985.

[13] Hristo Smolenov, Zeno's Paradoxes and Temporal Becoming, Studia Logica XLIII, D. Reidel Publishing Company, 1984.

[14] Hristo Smolenov, Sharing Genius with the Universe, The Light Second Code and the Golden Ratio, Sofia, 2016.

[15] Marija Gimbutas, The Language of the Goddess, Thames and Hudson, 2006; also: Maria Gimbutas, Gold Treasure at Varna. Archaeology, 30, (1977).

[16] John Baez, How Many Fundamental Constants Are There? Math.ucr.edu/home/baez/constants/html 